\title{
Simulating the effects of refractive index difference on the coupling efficiency of periodically segmented waveguide mode converter
}

\author{
Yu Zheng*, Hao He, Liangiong Jiang, Ji'an Duan \\ State Key Laboratory of High Performance Complex Manufacturing, \\ College of Mechanical and Electrical Engineering, Central South University, \\ Changsha, Hunan, 410083, China \\ ${ }^{*}$ Corresponding author: zhengyu@csu.edu.cn
}

\begin{abstract}
Efficient coupling of micro/nano-optical waveguides with single-mode fibers is the premise for the efficient operation of the integrated photonic chip, which directly determines its optical performance. In this paper, the design principles of periodically segmented waveguide (PSW) structure used for high-efficiency fiber-chip coupling are proposed, and the effects of refractive index difference $\Delta$ on coupling efficiency and structural parameters are studied by simulation. It is found that as the $\Delta$ of the PSW increases, the period of the PSW tends to be smaller, and the coupling efficiency decreases continuously, reduced by around $0.673 \mathrm{~dB}$ in the range of $\Delta=3 \%$ to $\Delta=7 \%$. Through the analysis of PSW optical mechanisms, it demonstrates that the main reason for the decrease of coupling efficiency is that the transmission loss of the tapered section increases sharply with the increase of $\Delta$. High- $\Delta$ PSW is difficult to apply to highly integrated silica optical chips due to the unignorably insertion loss.
\end{abstract}

Keywords: coupling efficiency, silica waveguide, planar lightwave circuit, micro/nano-optical waveguide.

\section{Introduction}

Through integrating waveguides $[\underline{1}, \underline{2}]$, resonators $[\underline{3}, \underline{4}]$, arrayed waveguide grating [ $\underline{5}]$ and other optical processing components on the micro/nano-scale, the integrated photonic chip can realize low-power and small-size on-chip optical function integration. However, because of the small-size characteristics of the waveguide that connects with the optical components to achieve cooperative operation, the large dimensional difference between the fiber mode $[\underline{6}]$ and the waveguide mode $[\underline{7}, \underline{8}]$ makes their coupling difficult, and the inefficient signal transmission between the fiber and the chip becomes a key problem which restricts the performance of the integrated photonic chip. To match the fiber mode with the waveguide mode, a mode conversion structure is usually required in the chip space. These structures are usually simple in form, but each 
has its own manufacturing process, size and coupling efficiency limitations. As a typical mode converter, a tapered waveguide [9] is commonly used in such structures. When the waveguide approaches the fiber end in the chip space, since the width of the cross -section gradually decreases, the mode in the tapered waveguide gradually expands in the transmission direction, eventually the optical field forms a spot whose mode size and mode effective index match the fiber mode. For example, the strategy that adds a polymer [10-12] waveguide over the tapered waveguide was proposed to limit the overexpansion of the waveguide mode. The presence of the polymer to some extent reduces the coupling loss, but the coupler is easily damaged because of its weak physical strength. A three-dimensional tapered waveguide structure on a photonic crystal $[\underline{13}, 14]$ was proposed by BARCLAY et al. [15], which realizes an almost adiabatic transmission from the fiber to a photonic crystal waveguide. However, the grayscale lithography [16] used in this design is not a standard technology in the industry, which makes integrating the design into chips difficult and complicated.

The proposed periodic segmented waveguide (PSW) mode converter $[\underline{17}, \underline{18}]$ scheme can complete the mode conversion without using an additional process. Unlike the tapered waveguide structure, PSW changes the effective refractive index along the waveguide direction by duty cycle modulation instead of width modulation, and the effective index of waveguide mode near the fiber end is close to the fiber mode effective index. The solution has been proven to be able to convert waveguide modes with high efficiency. In general, a well-designed PSW structure can realize about $1 \mathrm{~dB}$ coupling efficiency. This is far better than the case using a surface grating coupler, e.g. the coupling efficiency of the grating coupler [19] structure achieved by optimizing the buffer layer is $-2.3 \mathrm{~dB}$, and the coupling efficiency of the coupler [20] using a triangle array of circular holes, apodization and optimal buffer thickness is only $-1.74 \mathrm{~dB}$. Some literatures have studied this technology, and basically use large numerical aperture or lens fiber to couple with the segmented waveguide, and the efficiency is relatively high. However, there have been few reports of direct coupling using standard single-mode fibers. The reports about the influence of refractive index difference $\Delta$ on the PSW's coupling efficiency and parameter characteristics are also relatively few. Here the $\Delta$ is defined by core refractive index $n_{\text {core }}$ and cladding refractive index $n_{\text {clad }}$, i.e. $\Delta=\left(n_{\text {core }}-n_{\text {clad }}\right) / n_{\text {core }}$. For an integrated photonics system of fixed materials, high- $\Delta$ waveguides can improve the integration of the chip. However, the increase of the $\Delta$ makes the single-mode cross -section of the waveguide smaller, and the characteristics of the mode change. At the same time, the geometric parameters and coupling efficiency of the PSW designed for this waveguide will also alter. For some performance parameters, it may have been reduced to a very low level where the device is unusable.

The purpose of this paper is to consider these problems. The specific design process of PSW structure is presented, and the effects of $\Delta$ on the performance parameters such as segment loss and coupling loss are analyzed. The limitations of the high- $\triangle$ PSW structural manufacturing process are also explored. This paper uses the finite difference beam propagation method (FD-BPM $[\underline{21}, \underline{22}])$ to carry out this process and agrees that 
the fiber used in this paper is a standard single-mode silica fiber with a refractive index difference $\Delta=0.36 \%$ and a core diameter of $8.2 \mu \mathrm{m}$. The FD-BPM uses finite difference methods to solve the parabolic or paraxial approximation of the well-known Helmholtz equation, which is suitable for light field transmission problems with a small or zero propagation angle.

\section{Design principles and rules}

The PSW structure can be roughly represented in Fig. 1. The original continuous straight waveguide is divided into a number of segments by etching, and each segment is followed by a part filled with the substrate material to form a period. In the part close to the fiber end, the period and the duty cycle did not change along the $z$-axis (the $z$-axis is shown in Fig. 1), which means that the average refractive index of the segmented waveguide facing the fiber end remains the same, this section is called $\mathrm{I} / \mathrm{O}$ section. Away from the fiber end, the average refractive index changes to guide the lateral thinning of the mode, and the duty cycle of each period of this section stepwise changes along the $z$-axis. This part is referred to as a tapered section due to the presence of a duty cycle taper. They can be fabricated based on the same single-step lithography technique [23-27], but follow different design rules. In the I/O section, the fiber mode evolves into I/O fundamental mode over a certain distance. By properly distributing the duty cycle, the effective refractive index of the I/O fundamental mode can be close to the effective refractive index of the fiber mode to achieve mode matching. In the tapered section, the segmented waveguide may vary in width. If there is no width change, the performance of the taper is a linear or nonlinear change of the duty cycle along the propagation path.
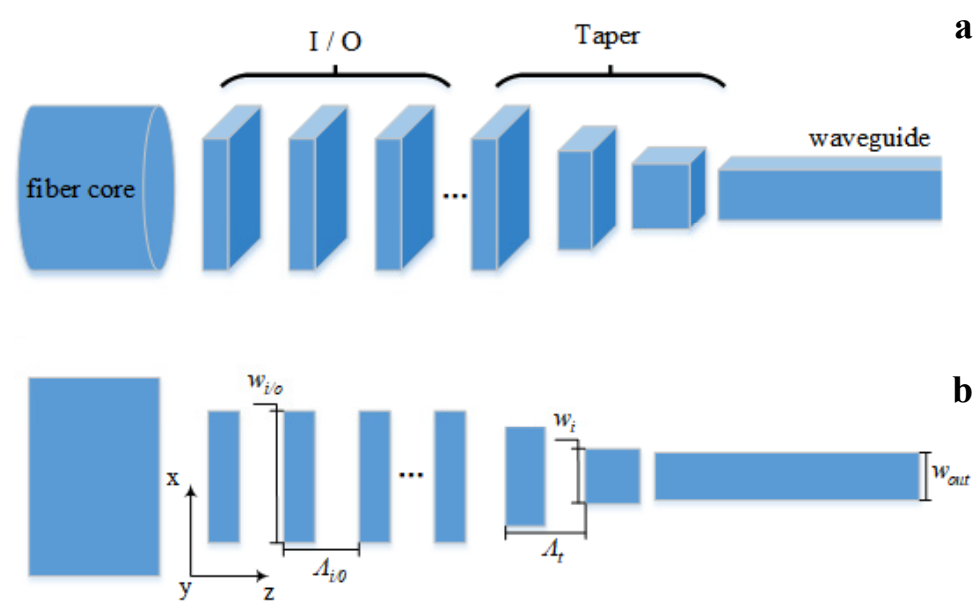

$\mathbf{a}$

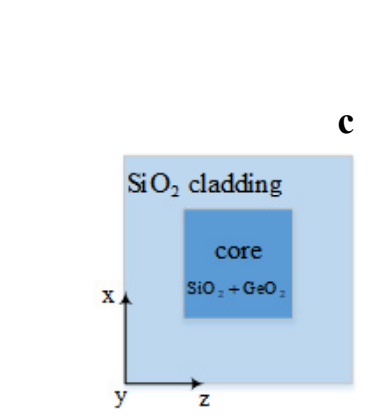

Fig. 1. General structure of PSW. (a) 3D side view of the PSW structure; (b) the in-plane views of the PSW structure; (c) material diagram of waveguide section, where $n_{\text {core }}>n_{\text {clad }}$, and the core material is $\mathrm{SiO}_{2}$ doped with small amount $\mathrm{GeO}_{2}$. 


\section{1. $\mathrm{I} / \mathrm{O}$ section}

The optimal duty cycle $\eta_{\mathrm{i} / \mathrm{o}}$ of the I/O section with a certain width must first be determined to minimize the mode mismatch between the $\mathrm{I} / \mathrm{O}$ section and the fiber. The process can be carried out in an equivalent manner. The I/O section can be regarded as a continuous waveguide with a smaller refractive index and equivalent refractive index contrast $\Delta n^{\prime}=\eta_{\mathrm{i} / \mathrm{o}} \Delta n$. Its mode overlap with the fiber can measure the mismatch loss. To calculate the overlap integral between the I/O fundamental mode and the fiber mode, the following formula [28] is used:

$$
O_{\mathrm{i} / \mathrm{o}}=\frac{\left[\iint \varphi_{1}(x, y) \varphi_{2}^{*}(x, y) \mathrm{d} S\right]^{2}}{\iint \varphi_{1}(x, y) \varphi_{1}^{*}(x, y) \mathrm{d} S \iint \varphi_{2}(x, y) \varphi_{2}^{*}(x, y) \mathrm{d} S}
$$

Since the waveguide is segmented, the periodic focusing and diffraction of the field in the $\mathrm{I} / \mathrm{O}$ section makes the transmission non-lossless. The segmentation loss makes the power of the I/O sectional mode exponentially decline along the path. And it can be characterized by the attenuation coefficient associated with the path $z$, calculated by $P_{\text {io }} / P_{\text {in }}=1-\alpha_{\mathrm{i} / \mathrm{o}} L_{\mathrm{i} / \mathrm{o}}$. Where $P_{\text {in }}$ is the optical power entering the I/O section, $P_{\text {io }}$ is the optical power output from the $\mathrm{I} / \mathrm{O}$ section, $L_{\mathrm{i} / \mathrm{o}}$ is the length of the $\mathrm{I} / \mathrm{O}$ section, $\alpha_{\mathrm{i} / \mathrm{o}}$ is the attenuation coefficient. Fiber mode can usually be converted to I/O fundamental mode in several I/O cycles, which means that the $\mathrm{I} / \mathrm{O}$ section is usually short and the $\alpha_{\mathrm{i} / \mathrm{o}}$ has little effect on the design of I/O section.

\subsection{Tapered section}

In the tapered section, the duty cycle and the width in each period can affect the size of the waveguide mode in the period. Compared with the case without the width taper, the existence of the width is equivalent to the enhancement of the refractive index difference in the corresponding period, it disturbs the refractive index of the period. The disturbance can be simply considered as the original refractive index multiply by a factor $W_{\mathrm{i}} / W_{\text {out }}$, $W_{\mathrm{i}}$ is the width of the $i$-th periodic waveguide segment of the tapered section, $W_{\text {out }}$ is the width of the output waveguide. Therefore, the refractive index difference of the $i$-th period of the tapered section can be determined by $\Delta n^{\prime}=\eta_{\mathrm{i}} \Delta n W_{\mathrm{i}} / W_{\text {out }}$. Actually, $\Delta n^{\prime}$ determines the average refractive index of the waveguide during the period. We make $\Delta n^{\prime}$ changing linearly to guide mode varying. For the width taper, it is simply set to be linear, as described in the following equations:

$$
\begin{aligned}
& W_{\mathrm{i}}=W_{\mathrm{i} / \mathrm{o}}+\frac{(i-1) \Lambda_{\mathrm{t}}}{L_{\mathrm{t}}}\left(W_{\text {out }}-W_{\mathrm{i} / \mathrm{o}}\right) \\
& \frac{W_{\mathrm{i}}}{W_{\text {out }}} \eta_{\mathrm{i}}=\frac{W_{\mathrm{i} / \mathrm{o}}}{W_{\text {out }}} \eta_{\mathrm{i} / \mathrm{o}}+\frac{(i-1) \Lambda_{\mathrm{t}}}{L_{\mathrm{t}}}\left(1-\frac{W_{\mathrm{i} / \mathrm{o}}}{W_{\text {out }}} \eta_{\mathrm{i} / \mathrm{o}}\right)
\end{aligned}
$$


where $\eta_{\mathrm{i}}$ is the duty cycle of the $i$-th period, $\Lambda_{\mathrm{t}}$ is the period of the tapered section, $L_{\mathrm{t}}$ is the total length of the tapered section. This setting means that the change in duty cycle is no longer linear along the direction of transmission when $W_{\mathrm{i} / \mathrm{o}} \neq W_{\text {out }}$. The duty cycle is gradually reduced from 1 at the waveguide end to the value of the I/O section, which ensures that the waveguide mode size continues to increase along the path toward the fiber. The presence of the width taper somewhat weakens the duty cycle modification of the mode size, slowing the rate of change of the duty cycle along the transmission direction.

\section{Results and discussion}

\subsection{Mismatch loss}

The simulation process is performed on the silica strip waveguide as shown in Fig. 1c. Silica has good dispersion performance and has an almost constant refractive index of about 1.444 at $\lambda=1550 \mathrm{~nm}$. The PSW structure for such waveguides is optimized using the above design rules. For a typical waveguide which $\Delta=2.5 \%$, the single-mode cross -sectional dimension is $3.5 \mu \mathrm{m} \times 3.5 \mu \mathrm{m}$ and its Gaussian-like mode field is tightly confined in the waveguide region. When segmenting the waveguide, the optical field starts to delocalize, the effective refractive index determined by width and duty cycle changes, which makes the mismatch loss vary. The I/O section mismatch loss of the PSW structure is shown in Fig. 2. There exists a duty cycle that minimizes the mismatch loss. As larger I/O section widths were used, the optimal duty cycle tends to a smaller value, and the overlap decreases at a smaller rate as the duty cycle increases. By using a $6 \mu \mathrm{m} \mathrm{I} / \mathrm{O}$ section width and segmenting the waveguide into $\eta_{\mathrm{i} / \mathrm{o}}=0.18$, the mismatch loss can be reduced to $0.069 \mathrm{~dB}$. In this process, the I/O section has only lateral width

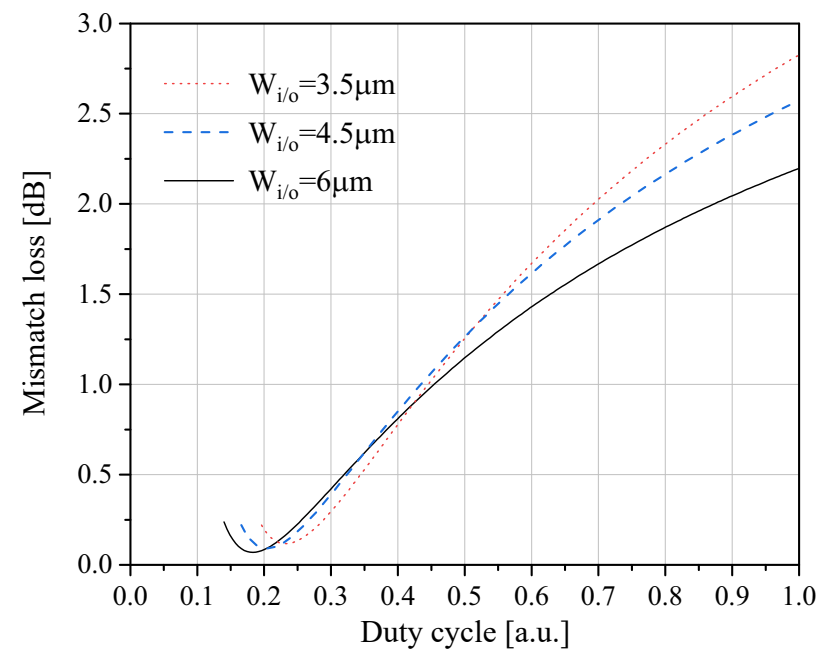

Fig. 2. Mode-overlap integral at the I/O section, as a function of duty cycle, with the width as a parameter, $\Delta=2.5 \%$. 


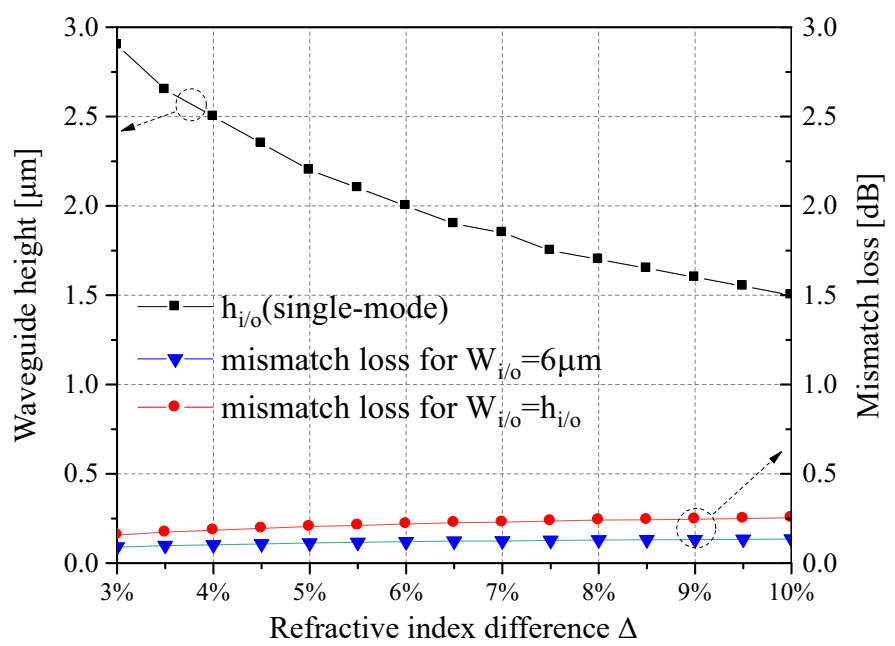

Fig. 3. Single-mode size and optimal overlap integral as a function of $\Delta$.

changes. The PSW structure described here has no longitudinal dimension change, and its depth is always the same as the waveguide single mode longitudinal dimension.

The optimal mode overlap value of the PSW structure under different $\Delta$ can be obtained in the same manner, and the optimal duty cycle corresponding to the optimal value is recorded. This process is carried out at a fixed wavelength $\lambda=1550 \mathrm{~nm}$ and $\Lambda_{\mathrm{i} / \mathrm{o}}=\lambda$, the results are drawn in Fig. 3. From the figure, it is found that as $\Delta$ increases, the single-mode size, i.e. waveguide size decreases, the optimal mode overlap value decreases, and the process is nearly linear. Considering the influence of the I/O section width, the case $\left(W_{\mathrm{i} / \mathrm{o}}=6 \mu \mathrm{m}\right)$ calculated in this paper finds that the existence of the I/O section width reduces the mismatch loss of the I/O section and needs a smaller duty cycle, which is consistent with the conclusion in Fig. 2. Larger width is also available, but it is necessary to pay attention to avoid the loss caused by multimode waveguide. Under different widths, the mismatch loss is less affected by $\Delta$, and the mismatch loss increases by only about $0.093 \mathrm{~dB}$ in the range of $\Delta=3 \%$ to $\Delta=10 \%$.

\subsection{Segmentation loss}

The segmentation loss can be reduced by assigning the I/O section period $\Lambda_{\mathrm{i} / \mathrm{o}}$ to an optimal value. Figure 4 shows the variation of $\alpha_{\mathrm{i} / \mathrm{o}}$ with the I/O section period. It can be seen from the figure that the segmentation loss keeps relatively low when using small $\Lambda_{\mathrm{i} / \mathrm{o}}$, corresponding to a smaller $\alpha_{\mathrm{i} / \mathrm{o}}$. When PSW uses larger I/O section width, the segmentation loss behaves better overall on all periods. There exists an optimum $\Lambda_{\mathrm{i} / \mathrm{o}}$ in each width case. Considering that the length of the I/O section can be short, it is reasonable to take a certain range around the optimal value.

Figure 5 depicts the optimal $\alpha_{\mathrm{i} / \mathrm{o}}$ at different $\Delta$. Two typical cases are considered in the simulation, the $\mathrm{I} / \mathrm{O}$ section width is the same as the width of output waveguide and the $\mathrm{I} / \mathrm{O}$ section width is close to the fiber core size (the width is $6 \mu \mathrm{m}$ ). As a result, 


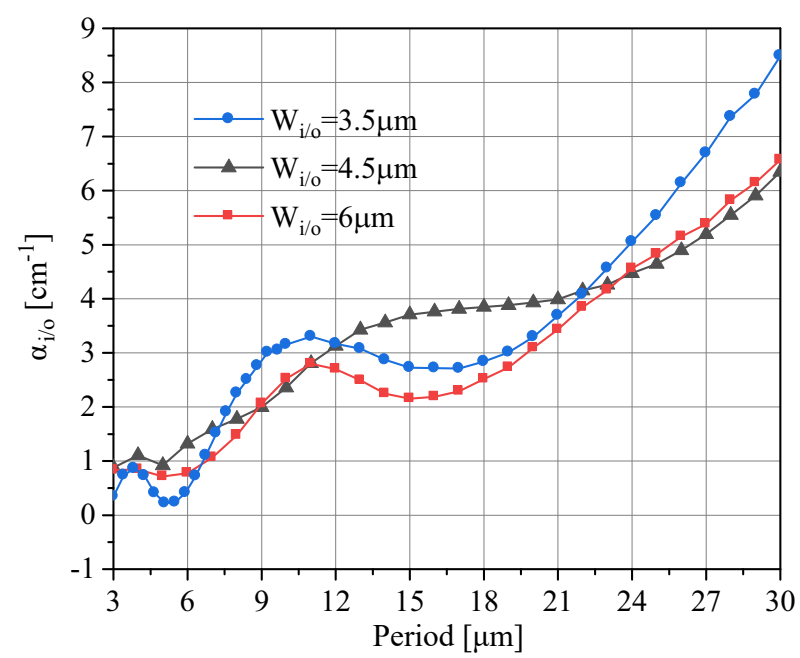

Fig. 4. The attenuation coefficient varies with the I/O section period for different cross-section dimensions, $\Delta=2.5 \%, \eta_{\mathrm{i} / \mathrm{o}}$ is the optimal value for the corresponding width.

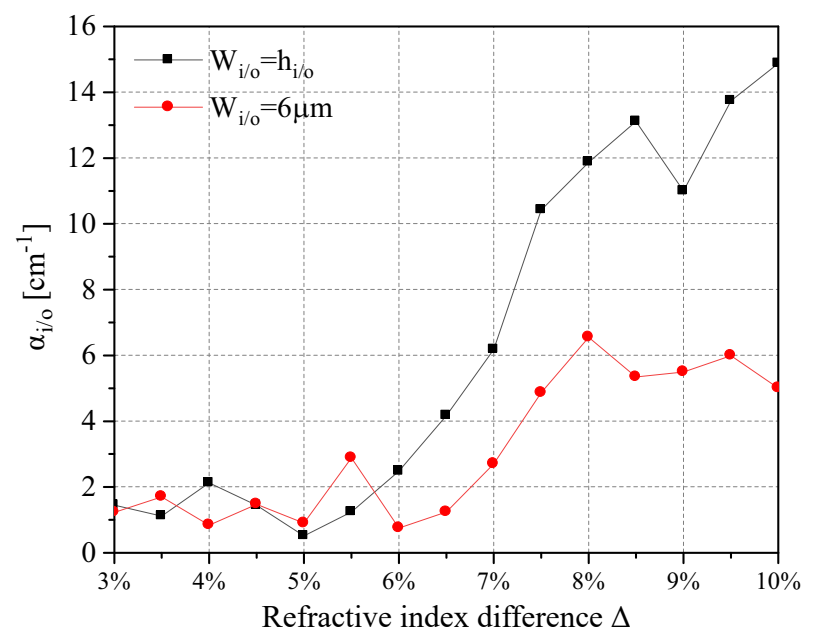

Fig. 5. Optimal attenuation coefficient at different $\Delta$, using the optimal duty cycle and period for the corresponding situation.

it was found that the $\alpha_{\mathrm{i} / \mathrm{o}}$ fluctuating rises with the increase of $\Delta$, and it increases sharply after $5.5 \%$. The case of the width taper is better at high $\Delta$ and has a lower $\alpha_{\mathrm{i} / \mathrm{o}}$. When the value of $\Delta$ is low, existing the width taper or not has little influences on the segment loss. After $\Delta=5.5 \%$, the segmentation loss has been very significant, even if a short $\mathrm{I} / \mathrm{O}$ section length is used, such as a length of $100 \mu \mathrm{m}$, the segmentation loss is more than about $0.22 \mathrm{~dB}$. This means that when the PSW uses a longer structure for the slower transition of the field mode size, its significant segmentation loss will be unavoidable. 


\subsection{Transmission efficiency in the tapered section}

For the tapered section, the parameters affecting its transmission efficiency are mainly the length and the period. Figure 6 shows the transmission efficiency of the tapered section as a function of period for different $\Delta$, it does not use a width taper and the value is converted to $\mathrm{dB}$ by $10 \log \left(f_{\text {transmission }}\right)$. At different $\Delta$, the initial duty cycle of the tapered section is the optimal value for the corresponding $\mathrm{I} / \mathrm{O}$ section. As the figure shown, for a fixed $\Delta$, the transmission efficiency fluctuating declines as the period increases. For a large refractive index difference, the period of the curve fluctuation is smaller and the transmission efficiency declines more rapidly. For $\Delta=6 \%$, the transmission efficiency is abruptly reduced to close to 0 in a period of $10 \mu \mathrm{m}$. When using a smaller period close to $\Lambda_{\mathrm{t}}=1 \mu \mathrm{m}$, the transmission efficiency under different $\Delta$ can reach more than $1 \mathrm{~dB}$. Simultaneously, it can be seen from the figure that the transmission efficiency of the fixed-length tapered section decreases as the refractive index difference increases. For the case of $\Delta=5 \%$, the transmission efficiency of the tapered section has been reduced to about $80 \%$. And the corresponding tapered section period under the optimal transmission efficiency tends to decrease as $\Delta$ increases, the curve which changes around the optimal transmission efficiency also gradually changes from flat to steep. This means that the well-designed high- $\Delta$ PSW taper section not only has a higher transmission loss but also has higher requirements for lithography precision used in manufacturing, and the loss due to process error is also greater.

Length is a primary factor in determining tapering loss. To evaluate this effect, the taper transmission efficiency was calculated, for $\Lambda_{\mathrm{t}}=1 \mu \mathrm{m}, W_{\mathrm{i} / \mathrm{o}}=6 \mu \mathrm{m}$. Due to the presence of the width taper, the duty cycle of the segment in the tapered section gradually decreases from the fiber end to the waveguide end and sets by Eq. (3). The results

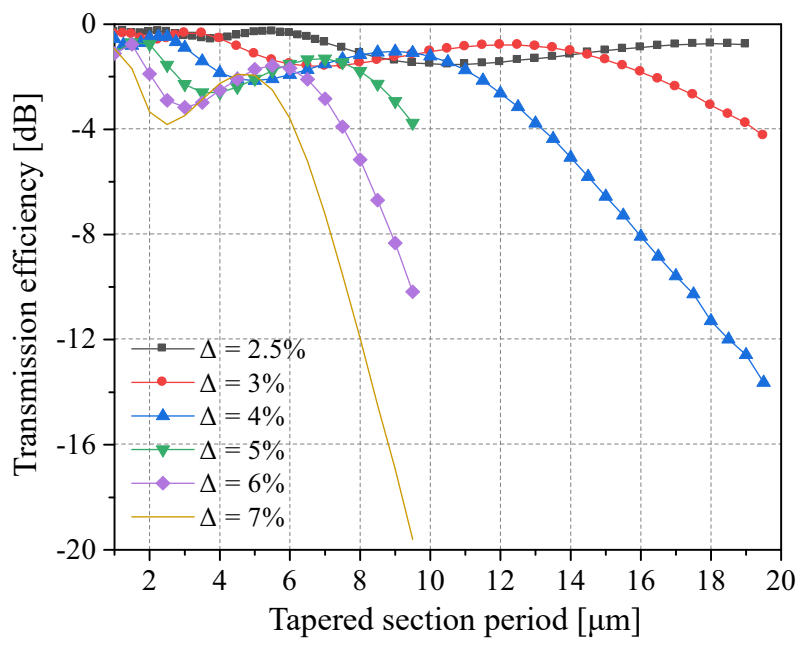

Fig. 6. Transmission efficiency of the tapered section as a function of the taper period, with the $\Delta$ as a parameter. The length of the tapered section is $500 \mu \mathrm{m}, W_{\mathrm{i} / \mathrm{o}}=6 \mu \mathrm{m}$. 


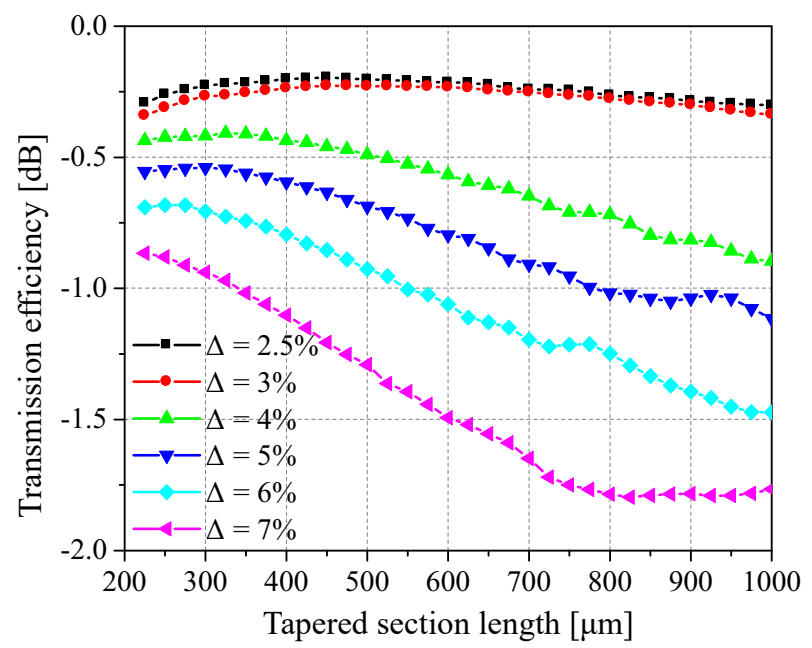

Fig. 7. Transmission efficiency of the tapered section, as a function of the length of the tapered section, with $\Delta$ as a parameter, $\Lambda_{\mathrm{t}}=1 \mu \mathrm{m}, W_{\mathrm{i} / \mathrm{o}}=6 \mu \mathrm{m}$.

of Fig. 7 show that when the length of the tapered section gradually increases, on the one hand, the stepped average refractive index changes more slowly, the mode transition loss during each period gradually decreases, and on the other hand, the number of segments increases for the same period, segmentation loss continues to increase. These two mechanisms determine the optimal value of the tapered length. When $\Delta$ is gradually increased, the influence of the segmentation loss gradually prevails, resulting in that the optimal taper length shifts to the left, and the transmission efficiency is at-

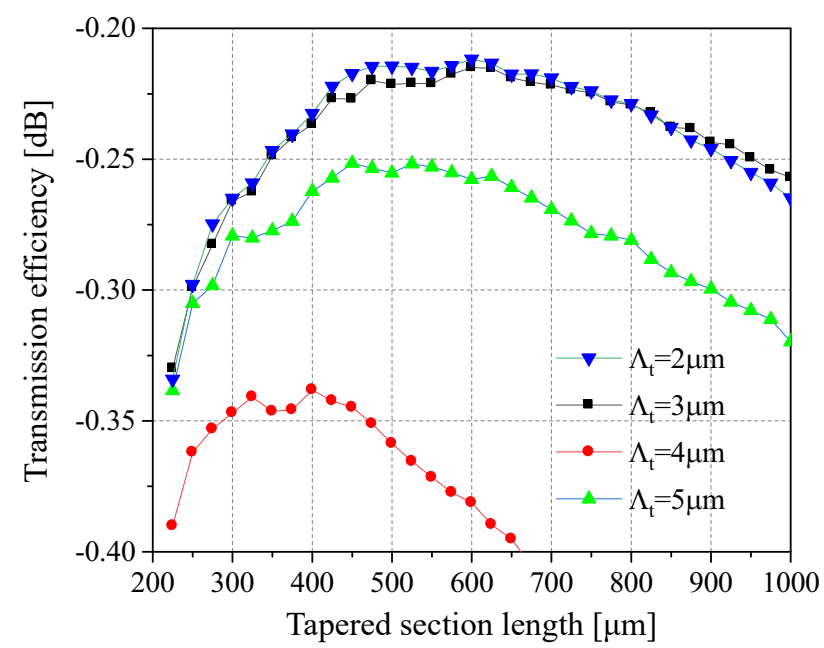

Fig. 8. Transmission efficiency of the tapered section as a function of the length of the tapered section, with the tapered period as a parameter, $\Delta=2.5 \%, W_{\mathrm{i} / \mathrm{o}}=6 \mu \mathrm{m}$. 
tenuated faster with the increase of the length. The optimal values of the taper length are different under different taper periods, but the rule that transmission efficiency increases first and then decreases with the increase of the taper length remains, which can be proved by Fig. 8. Compared to Fig. 7, because of the using of a relatively small refractive index difference, the transmission loss shown in Fig. 8 is smaller.

The PSW structure with perfect design parameters can achieve high efficiency coupling between the fiber and the waveguide. Figure 9 shows that the PSW structure designed for $\Delta=2.5 \%$ waveguide can achieve an over $90 \%$ efficient coupling. The same efficiency can also be obtained by using traditional tapered waveguide, but a fairly long transition structure is required, especially when $\Delta$ is high. As $\Delta$ increases, the total loss of the PSW becomes stronger, and the insertion loss will achieve $0.9 \mathrm{~dB}$ at $\Delta=6 \%$. Although PSW used perfect parameters, the coupling efficiency reduces around $0.673 \mathrm{~dB}$ from $\Delta=3 \%$ to $\Delta=7 \%$. In the design of the PSW structure, when trying to increase the device integration by increasing $\Delta$, on the one hand, the mismatch loss, segmentation loss, and taper loss of the PSW structure increase with the increase of $\Delta$, resulting in a decrease in coupling efficiency. Among them, the mismatch loss contributes less to this process, the segmentation loss has a greater influence and indirectly increases the loss of the tapered section. On the other hand, the optimal duty cycle, period and other design parameters of the PSW structure tends to be a smaller value

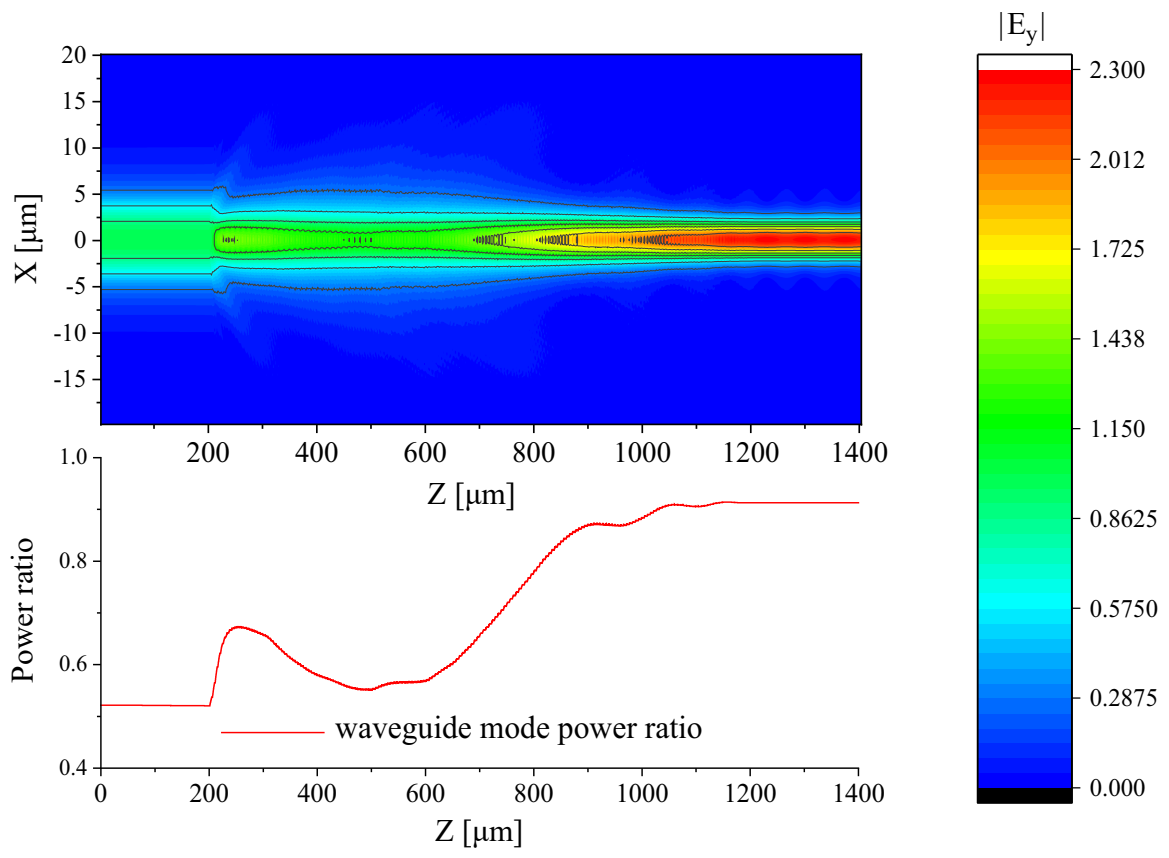

Fig. 9. PSW structure output optical power fraction relative to input power, the value is calculated in a rectangular area, the same size as the output waveguide mode, $W_{\mathrm{i} / \mathrm{o}}=3.5 \mu \mathrm{m}, \eta_{\mathrm{i} / \mathrm{o}}=0.23, L_{\mathrm{i} / \mathrm{o}}=300 \mu \mathrm{m}$, $\Lambda_{\mathrm{t}}=5.5 \mu \mathrm{m}, L_{\mathrm{t}}=700 \mu \mathrm{m}, \Delta=2.5 \%$. 
following the $\Delta$ increasing, which causes manufacturing difficulties and large process errors, making the structure difficult to integrate in high- $\Delta$ silica chips.

\section{Conclusion}

In this paper, the design rules of PSW structure are proposed. The influence of refractive index difference $\Delta$ on PSW coupler is studied by simulation. It is found that the well-designed PSW coupler can achieve highly efficient coupling between the fiber and the waveguide. However, as the system $\Delta$ increases, the coupling efficiency decreases. When the $\Delta$ increases to $6 \%$, the insertion loss has exceeded $0.9 \mathrm{~dB}$. In the simulation, it is found that the loss mainly occurs in the tapered section. When the $\Delta$ is small, the loss is extremely low. As the $\Delta$ increases, the transmission efficiency of the tapered section decreases sharply. The taper loss is the main section of the high- $\Delta$ PSW structure's loss. The analysis of the parameters such as the period, length and the transmission efficiency of the PSW structure shows that the increased manufacturing precision and the decreased coupling efficiency are the main reasons why the high- $\Delta$ silica chips are difficult to use the PSW technology. For the design of high- $\Delta$ fiberchip couplers, the PSW structure with a small period and short tapered section is suggested. In this paper, the width change of the tapered section is assumed to be linear, the use of non-linearity such as exponential or quadratic functional width changes may be effective in reducing coupling loss, which requires further simulation to determine. In addition, with the progress of lithography technology, new methods such as subwavelength or multilayer structure are expected to solve high- $\Delta$ fiber-chip coupling problems.

Author contributions: Conceptualization, Y.Z. and H.H.; methodology, C.W.; software, H.H.; validation, L.J., Y.Z. and H.H.; formal analysis, H.H.; investigation, Y.Z.; resources, H.H.; data curation, H.H.; writing - original draft preparation, H.H.; writing - review and editing, L.J.; visualization, C.W.; supervision, J.D.; project administration, Y.Z. and J.D.; funding acquisition, Y.Z. and J.D. All authors have read and agreed to the published version of the manuscript.

Funding: Supported by the National Natural Science Foundation of China (Grant No. 52175445), the State Key Laboratory of High Performance Complex Manufacturing, Central South University (Grant No. ZZYJKT2020-09), and the Natural Science Foundation of Hunan Province, China (Grant No. 2020JJ4247).

\section{References}

[1] Erol A.E., Sozuer H.S., High transmission through a $90^{\circ}$ bend in a polarization-independent single -mode photonic crystal waveguide, Optics Express 23(25), 2015, pp. 32690-32695, DOI: 10.1364/ OE.23.032690.

[2] Zheng Y., Gao P.P., Xiao Z.X., Zhou J.Y., Duan J.A., Chen B., Improving the lot fabrication stability and performance of silica optical films during PECVD, Applied Sciences 9(4), 2019, article 785, DOI: $10.3390 / a p p 9040785$.

[3] JiAo X.Q., Yu H.B., Yu M., XuE C.Y., ReN Y.F., Coupled resonator-induced transparency on a three -ring resonator, Chinese Physics B 27(7), 2018, article 074212, DOI: 10.1088/1674-1056/27/7/074212. 
[4] Bahadori M., Nikdast M., Rumley S., Dai L.Y., Janosik N., Van Vaerenbergh T., Gazman A., Cheng Q., Polster R., Bergman K., Design space exploration of microring resonators in silicon photonic interconnects: impact of the ring curvature, Journal of Lightwave Technology 36(13), 2018, pp. 2767-2782, DOI: 10.1109/JLT.2018.2821359.

[5] Zheng Y., Wu X.H., Jiang L.Q., Wu Y., DuAn J.A., Design of 4-channel AWG multiplexer/demultiplexer for CWDM system, Optik 201, 2020, article 163513, DOI: 10.1016/j.ijleo.2019.163513.

[6] Zheng Y., Li J.P., Gao P.P., DuAn J.A., Chen B., Packaging experiments of arrayed waveguide grating, Optik 168, 2018, pp. 179-183, DOI: 10.1016/j.ijleo.2018.04.105.

[7] Zheng Y., XIA B.X., High precision Fast Line Detection of alignment and coupling for planar Optical Waveguide device, Optik 145, 2017, pp. 519-528, DOI: 10.1016/j.ijleo.2017.08.040.

[8] Kwon M.S., Shin S.Y., Simple and fast numerical analysis of multilayer waveguide modes, Optics Communications 233(1-3), 2004, pp. 119-126, DOI: 10.1016/j.optcom.2004.01.037.

[9] Shoji T., Tsuchizawa T., Watanabe T., Yamada K., Morita H., Low loss mode size converter from 0.3 um square Si wire waveguides to singlemode fibres, Electronics Letters 38(25), 2002, pp. 1669 -1670 , DOI: $10.1049 / \mathrm{el}: 20021185$.

[10] Он M.C., Chu W.S., Shin J.S., Kim J.W., Kim K.J., Seo J.K., Lee H.K., Noh Y.O., Lee H.J., Polymeric optical waveguide devices exploiting special properties of polymer materials, Optics Communications 362, 2016, pp. 3-12, DOI: 10.1016/j.optcom.2015.07.079.

[11] Khan M.U., Justice J., Petaja J., Korhonen T., Boersma A., Wiegersma S., Karppinen M., Corbett B., Multi-level single mode 2 D polymer waveguide optical interconnects using nano-imprint lithography, Optics Express 23(11), 2015, pp. 14630-14639, DOI: 10.1364/OE.23.014630.

[12] YASUHARA K., Yu F., LSHIGURE T., Circular core single-mode polymer optical waveguide fabricated using the Mosquito method with low loss at 1310/1550 nm, Optics Express 25(8), 2017, pp. 8524-8533, DOI: $10.1364 / \mathrm{OE} .25 .008524$.

[13] Dudley J.M., Genty G., Coen S., Supercontinuum generation in photonic crystal fiber, Reviews of Modern Physics 78(4), 2006, pp. 1135-1184, DOI: 10.1103/RevModPhys.78.1135.

[14] Von Freymann G., Kitaev V., Lotsch B.V., Ozin G.A., Bottom-up assembly of photonic crystals, Chemical Society Reviews 42(7), 2013, pp. 2528-2554, DOI: 10.1039/c2cs35309a.

[15] Barclay P.E., Srinivasan K., Borselli M., Painter O., Experimental demonstration of evanescent coupling from optical fibre tapers to photonic crystal waveguides, Electronics Letters 39(11), 2003, pp. 842-844, DOI: 10.1049/el:20030565.

[16] Luo N., Zhang Z., Fabrication of a curved microlens array using double gray-scale digital maskless lithography, Journal of Micromechanics and Microengineering 27(3), 2017, article 035015, DOI: 10.1088/1361-6439/aa596a.

[17] Weissman Z., Hendel I., Analysis of periodically segmented waveguide mode expanders, Journal of Lightwave Technology 13(10), 1995, pp. 2053-2058, DOI: $10.1109 / 50.469728$.

[18] Cheben P., Schmid J.H., Wang S., Xu D.-X., Vachon M., Janz S., Lapointe J., Painchaud Y., PICARD M.-J., Broadband polarization independent nanophotonic coupler for silicon waveguides with ultra-high efficiency, Optics Express 23(17), 2015, pp. 22553-22563, DOI: 10.1364/OE.23.022553.

[19] Xu X., Subbaraman H., Covey J., Kwong D., Hosseini A., Chen R.T., Complementary metal-oxide -semiconductor compatible high efficiency subwavelength grating couplers for silicon integrated photonics, Applied Physics Letters 101(3), 2012, article 031109, DOI: 10.1063/1.4737412.

[20] Ding Y.H., Ou H.Y., Peucheret C., Ultrahigh-efficiency apodized grating coupler using fully etched photonic crystals, Optics Letters 38(15), 2013, pp. 2732-2734, DOI: 10.1364/OL.38.002732.

[21] Alcantara L.D.S., Teixeira F.L., Cesar A.C., Borges B.H.V., A new full-vectorial FD-BPM scheme: application to the analysis of magnetooptic and nonlinear saturable media, Journal of Lightwave Technology 23(8), 2005, pp. 2579-2585.

[22] SUJECKI S., Stability analysis of FD-BPM applied in high power semiconductor laser models, Optical and Quantum Electronics 47, 2015, pp. 1415-1419, DOI: 10.1007/s11082-014-0101-2. 
[23] Campbell M., Sharp D.N., Harrison M.T., Denning R.G., Turberfield A.J., Fabrication of photonic crystals for the visible spectrum by holographic lithography, Nature 404, 2000, pp. 53-56, DOI: $10.1038 / 35003523$.

[24] Fischer J., Wegener M., Three-dimensional optical laser lithography beyond the diffraction limit, Laser \& Photonics Reviews 7(1), 2013, pp. 22-44, DOI: 10.1002/lpor.201100046.

[25] D’Angelo M., Chekhova M.V., Shin Y., Two-photon diffraction and quantum lithography, Physical Review Letters 87(1), 2001, article 013602, DOI: 10.1103/PhysRevLett.87.013602.

[26] Donatini F., Dang L.S., A single-step electron beam lithography of buried nanostructures using cathodoluminescence imaging and low temperature, Nanotechnology 21(37), 2010, article 375303, DOI: $10.1088 / 0957-4484 / 21 / 37 / 375303$.

[27] Lee S., Jeong G.S., Kim J., Yoon J., Han S., Kang J.Y., Chung S., Lee S.H., Single-step UV diffraction lithography to define a hydrophobic SU-8 interconnected hoodoo structure, Microsystem Technologies 19, 2013, pp. 1025-1032, DOI: 10.1007/s00542-012-1693-8.

[28] Окамото K., Fundamentals of Optical Waveguide, Academic Press, America, New York, 2001, pp. 51-70.

Received September 15, 2020 in revised form November 7, 2020 